\title{
Efecto del momento de arrancado y la permanencia del cultivo en andana sobre el rendimiento, calidad lipídica y sanidad del grano de maní
}

\author{
Sebastian y Pérez, M., Gamba, J., Illa, C., Martín, M. P. y Pérez, M. A.
}

\begin{abstract}
RESUMEN
En el cultivo de maní su crecimiento indeterminado y la fructificación subterránea característica dificulta la identificación de las prácticas de cosecha para alcanzar el mayor rendimiento y calidad de grano. El objetivo de este trabajo fue evaluar el efecto del momento de arrancado y la posterior permanencia en andana sobre el rendimiento, calidad lipídica y sanidad del grano de maní. Los ensayos se realizaron con el cv. 'Granoleico' durante cuatro campañas. El diseño experimental fue completamente aleatorizado con tres repeticiones, con dos momentos de arrancado (150 y 165 días desde la siembra) y en cada uno de ellos, cuatro momentos de recolección $(7,14,21$ y 28 días desde arrancado). De acuerdo a los resultados, el retraso en el momento de arrancado aumentó el rendimiento sin cambios en la granometría, ni en la fracción lipídica. Por otra parte, aumentar la cantidad de días en la andana no mejoró estas variables, pero sí disminuyó la calidad sanitaria. Extender el ciclo del cultivo y la permanencia de las vainas en el campo en las condiciones evaluadas, no mejoró la calidad del grano de maní destinado a consumo humano.
\end{abstract}

Palabras clave: Arachis hypogaea, cosecha, granos infectados, lípidos

Sebastian y Pérez, M., Gamba, J., Illa, C., Martín, M. P. and Pérez, M. A., 2021. Effect of digging dates and the crop stay in the field on the yield, lipid quality and health of the peanut kernel. Agriscientia 38 (2): 117-125

\section{SUMMARY}

The indeterminate growth habit of peanut and characteristic underground fruiting make it difficult to identify the harvesting practices to achieve the highest yield and kernel quality. The objective of this work was to evaluate the effect of the digging dates and the subsequent stay in the field on the yield, lipid quality and health of the peanut kernels. The tests were carried out with cv. 'Granoleic' during four seasons. The experimental design was completely randomized with three repetitions, with two digging moments (150 and 165 days from sowing) 
and in each of them four harvesting dates (7, 14, 21 and 28 days). According to the results, the delay in the digging dates increased the yield without kernel size and lipid fraction changes. On the other hand, increasing the number of days in the windrow did not improve these variables, but it did decrease the sanitary quality. Extending the crop cycle and the permanence of the pods in the field under the evaluated conditions did not improve the quality of the peanut kernel destined for human consumption.

Key words: Arachis hypogaea, harvest, infected kernels, lipids

Sebastian y Pérez, M. (ORCID: 0000-0002-5281-0561), Gamba, J. (ORCID: 0000-0002-3659-2672) e IIIa, C. (ORCID: 0000-00018026-2622): Universidad Nacional de Córdoba (UNC), Facultad de Ciencias Agropecuarias (FCA), Catedra de Biología Celular, Centro de Transferencia Calidad Agroalimentaria. Córdoba, Argentina. Martín M. P. (ORCID: 0000-0002-4060-8956): Universidad Nacional de Córdoba (UNC), Facultad de Ciencias Agropecuarias (FCA), Cátedra de Microbiología. Córdoba, Argentina. Pérez M. A. (ORCID: 0000-0001-8400-5084): Universidad Nacional de Córdoba (UNC), Facultad de Ciencias Agropecuarias (FCA), Centro de Transferencia Calidad Agroalimentaria. Córdoba, Argentina. Correspondencia a: mauriciosebastian@gmail.com

\section{INTRODUCCIÓN}

La producción de maní (Arachis hypogaea L.) en Argentina se concentra en la provincia de Córdoba, donde se llevan a cabo actividades económicas de alto impacto social. La cadena agroalimentaria adquiere importancia en la generación de empleo, con efectos multiplicadores sobre la economía provincial y nacional, favoreciendo el asentamiento y el desarrollo social (Aguilar, 2018). A fin de poder mantener el sistema manisero y hacerlo sustentable en el tiempo, se está produciendo en la actualidad un desplazamiento del área de siembra desde la zona núcleo en el sur, hacia el centro norte provincial por lo que se hace necesario evaluar las prácticas de manejo en el campo para elevar la producción y la calidad (Gamba et al., 2020).

El grano de maní posee una cantidad importante de proteínas (aproximadamente $30 \%$ ), hidratos de carbono $(9-10 \%)$ y elevado contenido de aceite (44-56\%) entre otros componentes beneficiosos para la salud (Hassand y Amed, 2012; Arioglu et al., 2018); lo que lo convierte en un alimento importante para la humanidad (Bakal y Arioglu, 2019). En particular, la composición porcentual de los ácidos grasos en la fracción lipídica resulta determinante de las características sensoriales y la capacidad de conservación de los granos de maní (Dean et al., 2020). Por esta razón es importante medir el contenido de ácidos grasos en el grano, como efecto de la implementación de ciertas prácticas de manejo en el campo.
Cabe destacar que el cultivo de maní presenta hábito de crecimiento indeterminado, por lo que las plantas producen muchas flores durante el ciclo del cultivo, pero solo entre el 15 al $20 \%$ llegan a convertirse en vainas maduras (Kaba et al., 2014). Si bien la formación continua de vainas aumenta con el período de crecimiento, la determinación del momento de cosecha es fundamental para lograr la máxima productividad y calidad (Jordan et al., 2016; Pereira dos Santos et al., 2013; Zuza et al., 2017). Al respecto, evaluaciones realizadas con maní de diferentes variedades en condiciones distintas a las de la provincia de Córdoba reportaron que el retraso en el momento de cosecha favorece el rendimiento y la composición lipídica (Canavar y Kaynak, 2013a y 2013b; Gulluoglu et al., 2016; Bakal y Arioglu, 2019) así como el grado de madurez de las vainas (Branch et al., 2010; Gulluoglu et al., 2016; Arioglu et al., 2018). Por otro lado,Young et al. (1982) informaron pérdidas en cantidad y calidad al postergar el momento de la recolección.

En el cultivo de maní, su morfología con geocarpos en estrecho contacto con el sustrato y las condiciones de producción, favorecen el desarrollo de hongos (Pérez et al., 2007). Actualmente la alta presión de enfermedades fúngicas atenta la sustentabilidad del sistema, con el riesgo de que estos fitopatógenos produzcan micotoxinas, transformando los granos en no aptos para su consumo (Cuggino y Pérez, 2015; Mendonça Kluczkovski, 2019). 
De lo expuesto se deduce la importancia de evaluar las prácticas de manejo del cultivo en el campo durante la etapa de cosecha en condiciones locales. No solo por su impacto en el rendimiento, sino también por la sanidad como indicador de inocuidad del maní como alimento.

El objetivo de este trabajo fue evaluar el efecto del momento de arrancado y la posterior permanencia en andana sobre el rendimiento, calidad lipídica y sanidad del grano de maní.

\section{MATERIALES Y MÉTODOS}

Los ensayos se llevaron a cabo en el Módulo Maní del Campo Escuela de la Facultad de Ciencias Agropecuarias, ubicado en camino a Capilla de los Remedios km 15,5 $\left(31^{\circ} 28^{\prime} 49,42\right.$ " S y $\left.64^{\circ} 0036^{\prime}, 04^{\prime \prime} \mathrm{O}\right)$ de la provincia de Córdoba, Argentina. La fecha de siembra fue el 15 de noviembre en cada una de las campañas evaluadas (2014-2015, 2015-2016, 2016-2017 y 2018-2019). Se trabajó con semillas de maní cv. 'Granoleico' provisto por Criadero El Carmen, con valores de germinación no inferiores al $85 \%$. Las parcelas fueron de cuatro surcos de $100 \mathrm{~m}$ de longitud a $70 \mathrm{~cm}$, con una densidad de $145 \mathrm{~kg} / \mathrm{ha}$ y una distribución de 16 semillas por metro lineal. Durante el ciclo del cultivo se implementaron las prácticas de manejo habitualmente usadas en lotes comerciales. El arrancado se realizó en dos momentos a los 150 y 165 días desde la siembra (DDS) con una arrancadora invertidora de cuatro surcos. El maní permaneció en la andana en el campo y se procedió a cosechar manualmente todas las vainas en $1 \mathrm{~m}^{2}$ de superficie, a los 7,14 , 21 y 28 días desde el arrancado (DDA). Las vainas fueron embolsadas y trasladadas al laboratorio donde fueron lavadas con agua corriente para eliminar restos de tierra y oreadas a temperatura ambiente $\left(20+/-2^{\circ} \mathrm{C}\right)$ durante $48 \mathrm{~h}$, hasta $15+/-2 \%$ de humedad.

Las variables evaluadas fueron:

Estado de madurez: se determinó por el método de raspado del exocarpo con hidrolavadora a presión, exponiendo la coloración del mesocarpo. Las vainas fueron categorizadas de acuerdo a su color (blancas, naranjas, marrones y negras) por observación visual (Pérez et al., 2004). Los resultados se expresaron en porcentaje de madurez alcanzado como resultado de la suma de vainas de color naranja, marrón y negras.

Rendimiento: se separaron manualmente los granos de maní de las vainas para cada tratamiento y repetición y se procedió a pesarlos.
Los resultados se expresaron en $\mathrm{g} \cdot \mathrm{m}^{-2}$

Calidad granométrica: los granos de maní de cada tratamiento y repetición se clasificaron mediante paso por zarandas (en planta modelo Vignatti 2001), obteniéndose los calibres correspondientes a granos confitería identificados como 38/42, 40/50, 50/60 y 60/70 granos por onza. Cada fracción fue pesada y los resultados expresados en porcentaje respecto al peso total de la muestra.

Contenido de aceite: a partir de los granos cosechados en cada momento de arrancado y recolección, se obtuvo el aceite por prensado en frío mediante prensa hidráulica de 20 toneladas (HE-DU S.R.L., Argentina). El contenido de lípidos totales se determinó por el Método Soxhlet Official Methods of Analysis (Association of Official Analytical Chemists [AOAC], 1990) y el contenido de ácido oleico por cromatografía líquida gaseosa (GLC, por sus siglas en inglés; AOAC, 2007). Se prepararon los ésteres metílicos de los ácidos grasos (EMAGs) por transmetilación con una solución de ácido sulfúrico en metanol (30 g/L) previa saponificación con $\mathrm{KOH}$ 0,5 N en metanol. Los EMAGs se analizaron en un cromatógrafo de gases Perkin Elmer Clarus 600 (EE. UU.) equipado con un detector de ionización de llama (FID, por sus siglas en inglés). Se usó una columna capilar SACTM-5 (30 m x 0,25 mm i.d., 0,25 $\mu \mathrm{m}$ espesor; C\#24156; Supelco, EE. UU.). El gas portador fue nitrógeno (1 $\mathrm{mL} / \mathrm{min})$ y se empleó el siguiente programa de temperaturas: temperatura inicial $180^{\circ} \mathrm{C}$, con un aumento de $4^{\circ} \mathrm{C} / \mathrm{min}$ hasta $240^{\circ} \mathrm{C}$ (10 min). Los tiempos de retención relativos se consideraron en relación con el del palmitato de metilo y el contenido del ácido graso oleico se expresó como valor porcentual con relación al contenido graso total (Asensio et al., 2015).

Sanidad: se evaluó de acuerdo al método de Blotter test modificado (Mathur y Kongsdal, 2003). Para cada tratamiento y repetición se sembraron 50 granos en cámara húmeda en condiciones controladas $\left(21^{\circ} \mathrm{C}\right.$ y $12 \mathrm{~h}$ luz UV). A los 7 días se determinó la incidencia fúngica con los principales patógenos de maní (Aspergillus flavus, Aspergillus niger, Fusarium spp., Penicillium spp.) mediante observaciones microscópicas y el uso de claves (Ellis, 1971; Mathur y Kongsdal, 2003). Los resultados se expresaron en porcentaje del total de granos infectados con los hongos mencionados.

Diseño experimental y análisis estadístico: Ios ensayos se realizaron según un diseño experimental completamente aleatorizado con tres repeticiones, con dos momentos de arrancado (150 y 165 DDS) y en cada uno de ellos cuatro momentos de 
recolección $(7,14,21$ y 28 DDA). Las variables se analizaron con modelos lineales generales mixtos y ANAVA para un modelo trifactorial con interacción entre los factores campaña *momento de arrancado*momento de recolección. Las medias se compararon mediante la prueba DGC (Di Rienzo et al., 2002) con un nivel de significación del $5 \%(p \leq 0,05)$ usando el software estadístico InfoStat (Di Rienzo et al., 2019).

\section{RESULTADOS Y DISCUSIÓN}

Los resultados de rendimiento obtenidos en este trabajo, no mostraron interacción triple entre los factores campaña*momento de arrancado*momento de recolección $(p=0,0789)$. Al retrasar 15 días el momento de arrancado (Figura 1) se incrementó significativamente el rendimiento de granos $(p=0,0001)$. Este comportamiento coincide con lo propuesto por Canavar y Kaynar (2013a) y Gullouglu et al. (2016), quienes informaron aumentos en los rendimientos al retrasar la cosecha en cultivares tipo Virginia. De acuerdo a lo sugerido por Young et al. (1982) y Williams (2000), la prolongación del ciclo del cultivo favorece la formación de flores y vainas debido a su característico crecimiento indeterminado, lo que conduce al aumento del rendimiento.

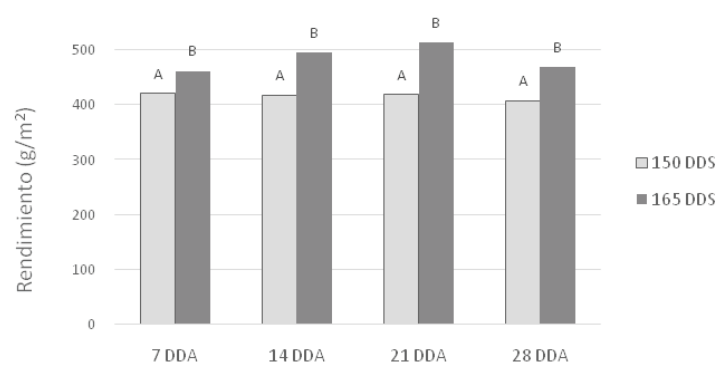

Figura 1. Rendimiento de granos de maní en diferentes momentos de arrancado (150 y 165 DDS) y recolección. Valores promedios de las campañas 2014-2015, 2015-2016, 2016-2017 y 2018-2019. Campo Escuela FCA UNC, Córdoba Argentina. Letras distintas indican diferencias significativas $(p<0,05)$

En la Figura 2 A y B, se presentan los porcentajes de cada fracción correspondientes a los diferentes momentos de arrancado (150 y 165 DDS). La proporción de granos de acuerdo al tamaño fue dependiente de la campaña analizada (Figura 2 C). La categoría Confitería $(38 / 42+40 / 50+50 / 60)$ en las campañas 2014-2015 y 2015-2016 no mostraron diferencias significativas al retrasar el momento de arrancado. Sin embargo, en los períodos evaluados 2016-2017 y 2018-2019 el porcentaje de granos

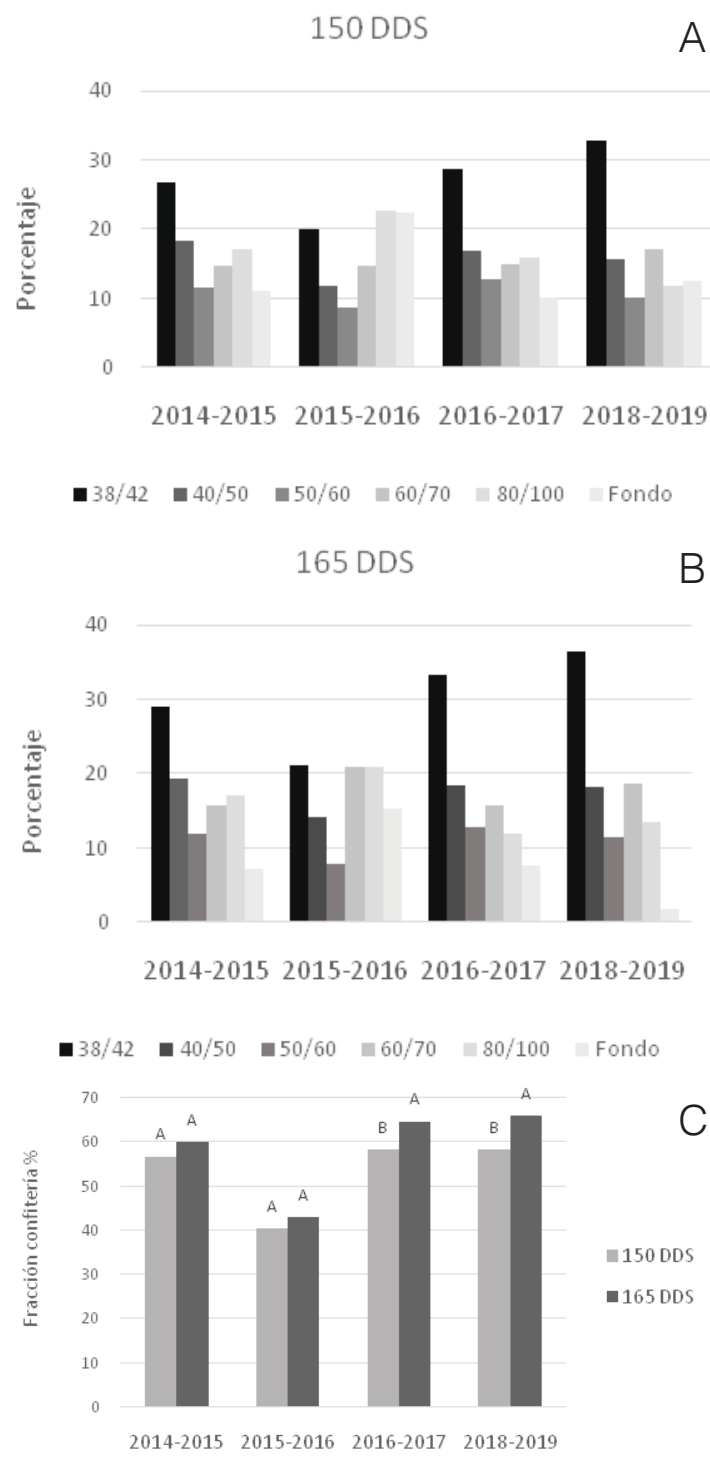

Figura 2. Categorías de acuerdo al tamaño de granos de maní provenientes de plantas arrancadas en diferentes momentos (150 y 165 DDS) en distintas campañas 2014-2015, 2015-2016, 20162017 y 2018-2019. Campo Escuela FCA UNC, Córdoba Argentina. Letras distintas indican diferencias significativas $(p<0,05)$. Letras distintas indican diferencias significativas $(p<0,05)$

Confitería fue mayor y se registró un aumento significativo del $9 \%$ al prolongar el momento de arrancado de 150 a 165 DDS. Los aumentos registrados en el tamaño comercial de granos, acuerdan con lo informado por Jordan et al. (2016) al evaluar el cv. 'Gregory' en las condiciones de Carolina del Norte, EE. UU. Al respecto, Zuza et al. (2017) no informaron variaciones en el tamaño de grano al retrasar 10 días la cosecha de maní tipo Spanish. Mientras que Ariouglu et al. (2018) en variedades tipo Virginia, hallaron aumento en 
el peso promedio de los granos sin considerar las variaciones en cada tamaño. Es importante destacar que el tamaño comercial alcanzado define el destino industrial y aquellas fracciones mayores, identificadas como maní apto para consumo, son mejor retribuidas económicamente al productor.

Otra de las características determinantes de la calidad del grano es el grado de madurez alcanzado. Los valores observados en las diferentes campañas (Figura 3A) variaron entre el 47 al $55 \%$ sin diferencias significativas entre ellas $(p \leq 0,05)$. Estos valores fueron superiores a los informados por Gamba et al. (2014), quienes reportaron porcentajes de madurez no mayores al $40 \%$ para
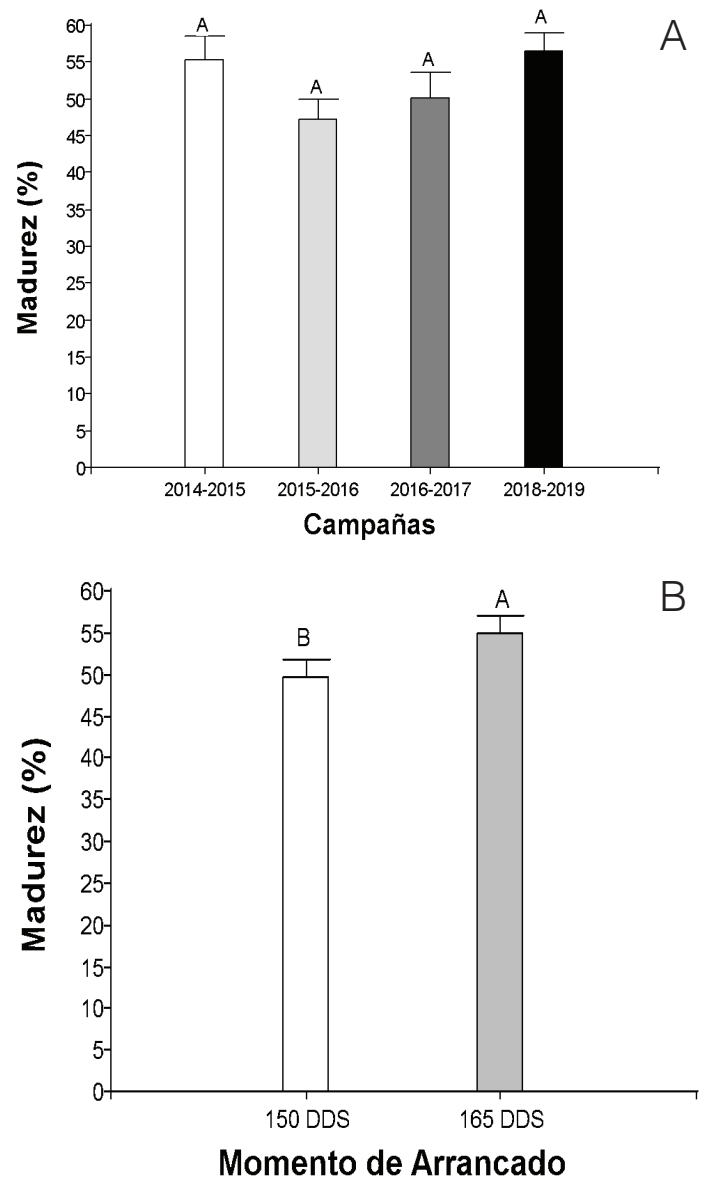

Figura 3. Madurez de vainas de maní (\%) en diferentes campañas (A) y valores promedios de madurez de vainas de maní (\%) para las campañas 2014-2015, 2015-2016, 2016-2017 y 20182019 según momento de arrancado (B). Campo Escuela FCA UNC, Córdoba Argentina. Letras distintas indican diferencias significativas $(p<0,05)$. variedades tipo Runner en las condiciones del sur de la provincia de Córdoba. Al respecto, Arioglu et al. (2018) no hallaron diferencias en el grado de madurez de vainas de maní tipo Virginia, entre las campañas evaluadas en el mediterráneo (Turquía).

En este trabajo, las condiciones ambientales durante las sucesivas campañas (Tabla 1) no presentaron variaciones importantes, en particular la acumulación térmica durante el ciclo del cultivo, determinante del porcentaje de madurez de las vainas de maní de acuerdo a lo propuesto por Gamba et al. (2014). Sin embargo, los resultados del grado de madurez alcanzado mostraron interacción $(p \leq 0.0185)$ por efecto de los factores campaña*momento de arrancado. El retraso en el momento de arrancado (Figura 3B) aumentó significativamente el porcentaje de vainas maduras en un $9,85 \%$. Este comportamiento ha sido registrado por Gullouglu et al. (2016) y Arioglu et al. (2018) en cultivares tipo Virginia. El incremento en el nivel de madurez de las vainas se explica por la mayor acumulación térmica durante el ciclo del cultivo (Rowland et al., 2006; Gamba et al., 2020) que al prolongarse por el retraso en el momento de recolección, beneficia la actividad anabólica sobre todo en la fracción lipídica (Canavar y Kaynak, 2013b). Se debe tener en cuenta que establecer el momento de arrancado solo sobre la base del máximo grado de madurez, no asegura la recolección del mayor número de vainas (Kaba et al., 2014). Esto debido a que, la máxima madurez no se vincula con la mayor resistencia del clavo (Gilmany Smith, 1977) provocando pérdidas por la retención de vainas en el suelo (Jordan et al., 2016).

Tabla 1. Valores promedio de temperatura ( ${ }^{\circ} \mathrm{C}$ día acumulados) y precipitaciones acumuladas $(\mathrm{mm})$ durante el ciclo del cultivo de maní en diferentes campañas. Campo Escuela FCA UNC, Córdoba Argentina

\begin{tabular}{lcc}
\hline Campaña & $\begin{array}{c}\text { Temperatura } \\
{ }^{\circ} \text { C día acumulados }\end{array}$ & $\begin{array}{c}\text { Precipitaciones } \\
\mathrm{mm}\end{array}$ \\
\hline $2014 / 2015$ & 1599,85 & 718 \\
$2015 / 2016$ & 1647,50 & 590 \\
$2016 / 2017$ & 1506,25 & 513 \\
$2018 / 2019$ & 1541,11 & 735 \\
\hline
\end{tabular}

Temperatura base para el cálculo de ${ }^{\circ} \mathrm{C}$ día: $11^{\circ} \mathrm{C}$ hasta el día 6 DDS, luego $13^{\circ} \mathrm{C}$ hasta el final del ciclo.

Respecto al contenido de lípidos totales (Figura 4) los valores promedio registrados se encuentran dentro de lo informado para el cv. 'Granoleico' (Ryan, 2011). Las condiciones en cada campaña no ocasionaron diferencias significativas $(p \leq 0,05)$; resultados similares fueron reportados por 

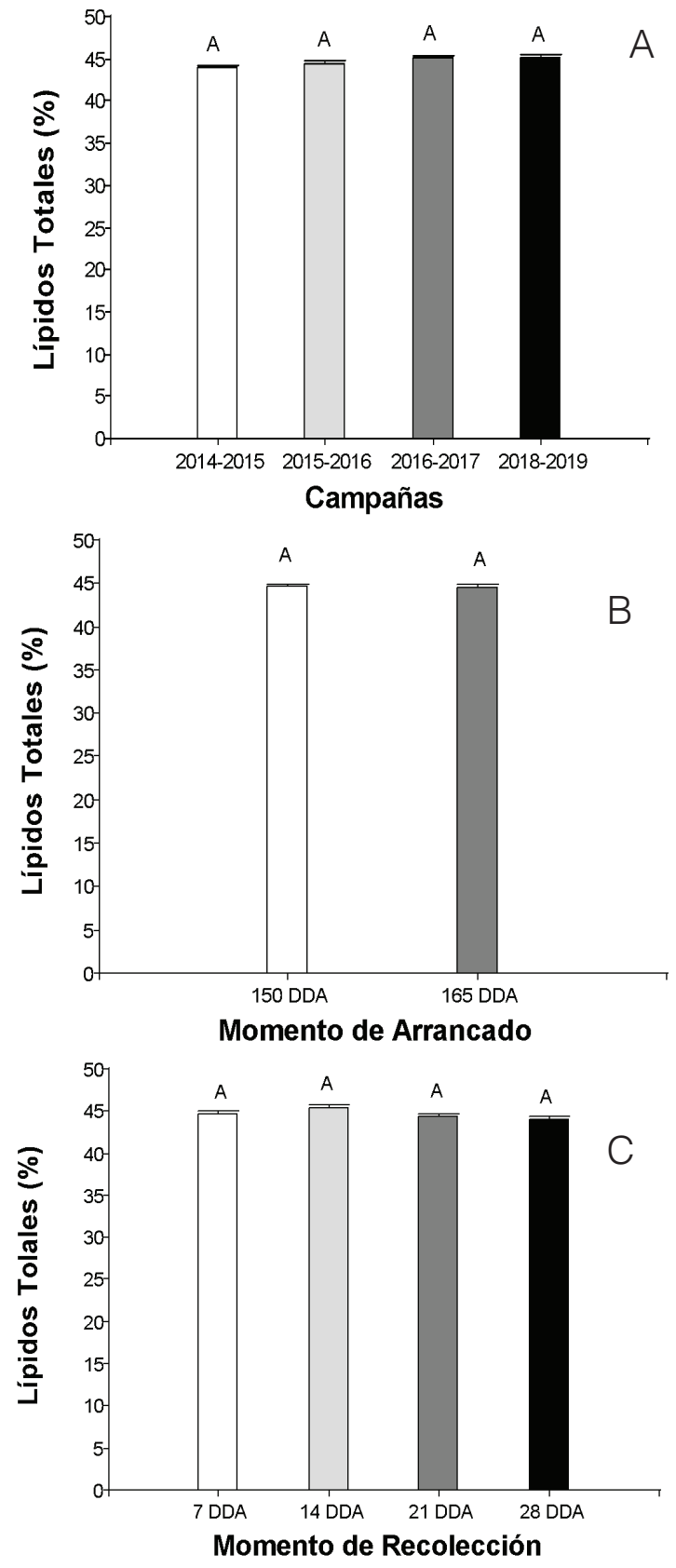

Figura 4. Contenido de lípidos totales (\%) en granos de maní en diferentes campañas (A), y valores promedios de lípidos totales (\%) de las campañas 2014-2015, 2015-2016, 2016-2017 y 20182019 según momento de arrancado (B) y recolección (C). Campo Escuela FCA UNC, Córdoba Argentina. Letras iguales indican diferencias no significativas $(p<0,05)$

Canavar y Kaynak (2013b) y Gullouglu et al. (2016). Además, no se observó interacción ( $p \leq 0,00398)$ entre los factores analizados (campaña * momento de arrancado*momento de recolección).
Coincidentemente, Canavar y Kaynak (2013b) en ensayos realizados con cultivares de maní tipo Virginia, no encontraron diferencias en el porcentaje de lípidos totales al retrasar el momento de recolección, pero sí en su composición. En particular, el contenido de ácido oleico registrado (Figura 5) superó a lo informado en estudios previos (Hassand y Ahmed, 2012; Onemli, 2012; Canavar y Kaynak, 2013b; Gullouglu et al., 2016). Esto es debido a que en el cv 'Granoleico' evaluado, los genes que codifican las enzimas desaturasas han mutado y, por lo tanto, no se lleva a cabo el proceso de transformación de ácido oleico en linoleico, razón por la cual se mantiene elevado el contenido de ácido graso oleico (Dean et al., 2020).

Se detectaron diferencias significativas en el porcentaje de oleico entre campañas (Figura $5 \mathrm{~A})$; los mayores valores se observaron en los períodos 2014-2015 y 2018-2019. Si bien la mayor acumulación térmica favorece la mayor proporción de oleico (Onemli, 2012), en las campañas evaluadas el factor ambiental diferencial fue la mayor disponibilidad hídrica durante los períodos críticos en el cultivo lo que determinó la acumulación de este ácido graso (Tabla 1).

Es de destacar que el contenido de lípidos totales (Figura 4) y en particular el porcentaje de ácido oleico (Figura 5), no mostraron diferencias significativas para los diferentes momentos de arrancado y recolección evaluados. Los resultados acerca de la composición de la fracción grasa sin variaciones se contraponen a lo informado por Canavar y Kaynak (2013b) y Gullouglu et al. (2016), quienes indicaron que el retraso en el momento de arrancado aumenta el contenido de ácido oleico en maní de la var. tipo Virginia.

Según los resultados presentados en este estudio, el aumento en el porcentaje de vainas maduras resultante del retraso del momento de arrancado, no estuvo relacionado con un mayor contenido de ácido oleico, contrariamente a lo propuesto por Canavar y Kaynak (2013a); Dean et al. (2020) y Gulluoglu et al. (2016) en ensayos realizados con variedades de maní tipo Virginia. De lo expuesto se deduce que, en las campañas evaluadas, la composición lipídica en los granos de maní presentó una relación estrecha entre el cultivar evaluado, la temperatura acumulada por sobre el requerimiento mínimo y la mayor disponibilidad hídrica. Debe destacarse que la fracción grasa está estrechamente relacionada con la calidad del grano de maní obtenido, en lo que se refiere a la estabilidad oxidativa y el valor sensorial de los productos derivados (Dean et al., 2020).

Respecto al estado sanitario, el porcentaje de 


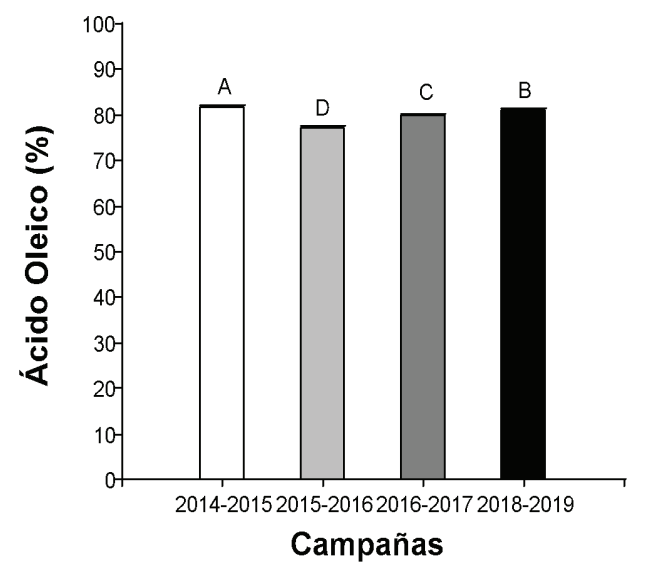

A
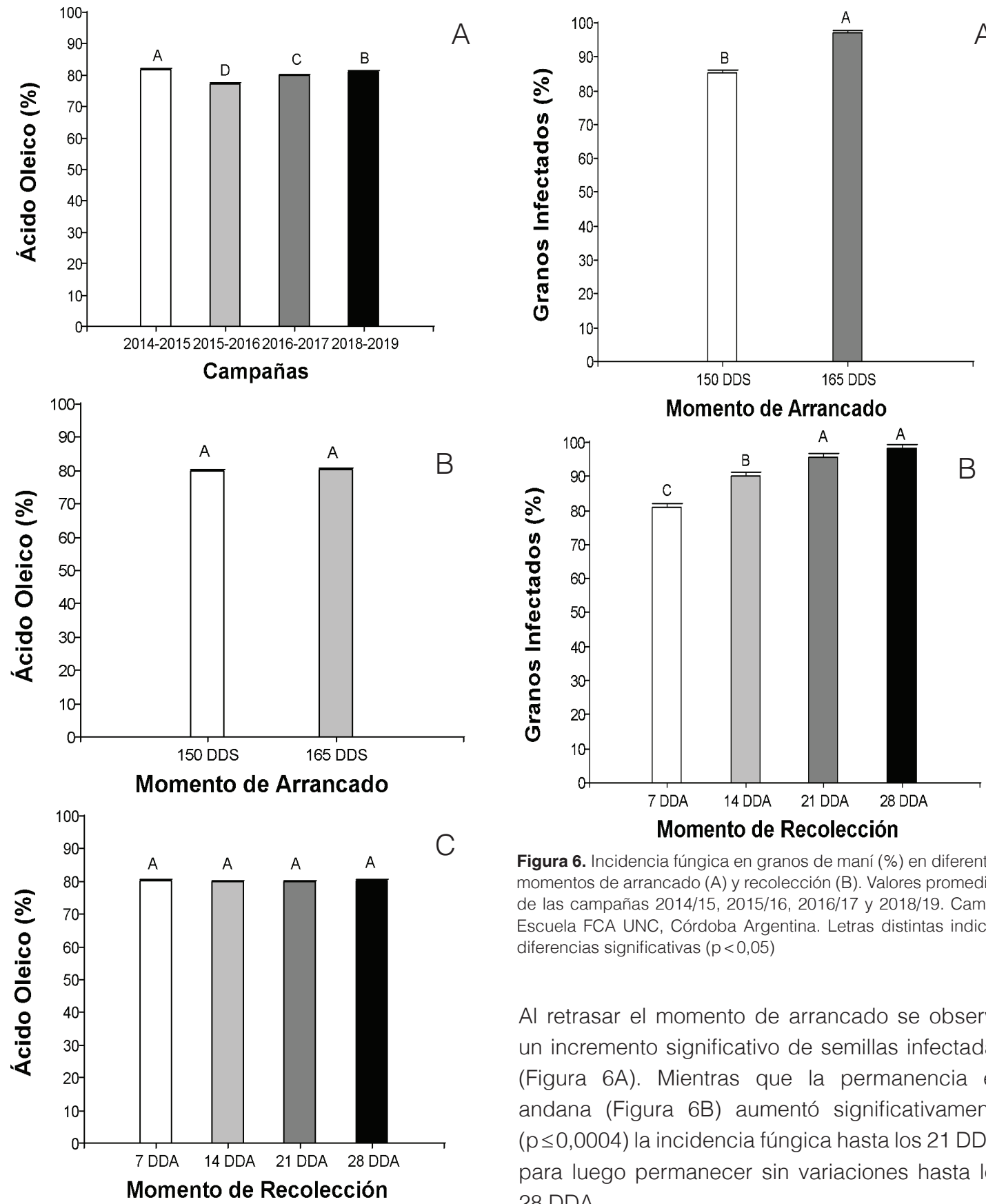

Figura 6. Incidencia fúngica en granos de maní (\%) en diferentes momentos de arrancado (A) y recolección (B). Valores promedios de las campañas 2014/15, 2015/16, 2016/17 y 2018/19. Campo Escuela FCA UNC, Córdoba Argentina. Letras distintas indican diferencias significativas $(p<0,05)$

Al retrasar el momento de arrancado se observó un incremento significativo de semillas infectadas (Figura 6A). Mientras que la permanencia en andana (Figura 6B) aumentó significativamente $(p \leq 0,0004)$ la incidencia fúngica hasta los 21 DDA, para luego permanecer sin variaciones hasta los 28 DDA.

La permanencia de las vainas de maní en

Figura 5. Contenido de ácido oleico (\%) en granos de maní en diferentes campañas (A), y valores promedios de ácido oleico (\%) de las campañas 2014-2015, 2015-2016, 2016-2017 y 20182019 según momento de arrancado (B) y recolección (C). Campo Escuela FCA UNC, Córdoba Argentina. Letras iguales indican diferencias no significativas $(p<0,05)$

granos infectados (Figura 6), no mostró interacción entre los factores campaña*momento de arrancado*momento de recolección $(p \leq 0,0001)$. el campo, expuestas a las condiciones medio ambientales favorece la infestación fúngica (Pérez et al., 2007) e inclusive aumenta la posibilidad de generar micotoxinas que disminuyen la calidad del grano de maní como alimento (Canavar and Kaynak, 2013b; Cuggino y Pérez, 2015; Mendonça Kluczkovski, 2019). 


\section{CONCLUSIONES}

El retraso de 15 días en el momento de arrancado incrementó significativamente el rendimiento de maní como consecuencia del aumento en el número de vainas y dependiendo de las condiciones ambientales en cada campaña puede aumentar el tamaño de granos correspondientes a la fracción confitería.

Si bien el retraso en el momento de arrancado incrementó el grado de madurez de las vainas, no aumentó el contenido de lípidos totales, ni el porcentaje de ácido graso oleico, ni siquiera al prolongar su permanencia en la andana.

La permanencia del cultivo en andanas no influyó en el aumento de rendimiento. Por el contrario, la mayor exposición a las condiciones ambientales en el campo favoreció la infección con hongos fitopatogénicos potenciales formadores de micotoxinas.

Por lo expuesto se deduce que, desde el punto de vista tecnológico, se hace necesario ajustar la logística para el arrancado según el cultivar, disminuyendo la permanencia en la andana, a fin de mantener las características del grano de maní logradas durante el ciclo del cultivo.

\section{BIBLIOGRAFÍA}

Aguilar, L. (2018). La ruta del maní. Todo Maní. http:// www.todomani.com.ar/mani/notas.asp?nid=1065

Association of Official Analytical Chemists (AOAC) (1990). Official methods of analysis (15a. ed.). Association of Analytical Chemistry.

Association of Official Analytical Chemists (AOAC) (2007). Official methods of analysis. Association of Official Analytical Chemists. 18 ${ }^{\text {th }}$ Edition.

Arioglu, H., Bakal, H., Gulluoglu, L., Onat B. y Kurt, C. (2018). The effect of harvesting dates on some agronomic and quality characteristics of peanut (Arachis hypogaea L.) varieties grown as a main crop in mediterranean region (Turkey). Turkish Journal of Field Crops, 23(1), 27-37. https://doi.org/10.17557/ tjfc. 414856

Asensio, C. M., Grosso, N. R. y Juliani, R. H. (2015). Quality preservation of organic cottage cheese using oregano essential oils. LWT - Food Science and Technology, 60(2), 664 - 671. https://doi.org/10.1016/j. Iwt.2014.10.054

Bakal, H. y Arioglu, H. (2019). The determination of fatty acids composition and oil quality factors of some peanut varieties having different market types at different harvesting times in main and double crop growing seasons in Mediterranean region. Turkish Journal of Field Crops, 24(2), 221-229. https:// doi. org/10.17557/tjfc.655078

Branch, W. D., Bostick, J. P., Williams, E. J. y Beasley, J. P. Jr. (2010). Determination of the relative maturity range for the 'Georgia-02C' peanut cultivar. Peanut Science, 37(2), 106-109. https://doi.org/10.3146/PS09-023.1

Canavar, O. y Kaynak, M. A. (2013a). Determination of yield and yield components and seed quality of peanuts (Arachis hypogaea L.) at different harvest times. International Journal. of Agronomy and Plant Production, 4, 3791-3803.

Canavar, O. y Kaynak, M. A. (2013b). Prevention of preharvest aflatoxin production and the effect of different harvest times on peanut (Arachis hypogaea L.) fatty acids. Food Additives \& Contaminants, 30(10), 18071818. http://dx.doi.org/10.1080/19440049.2013.81872 0

Cuggino, S. y Pérez, A. A. (2015). Assessment of the implementation degree in handling practices which contribute to reducing fungal incidence and its consequent aflatoxins production in peanut kernels. International Journal of Applied Science and Technology, 5(6), 143-149. https://www.ijastnet.com/ journals/Vol_5_No_6_December_2015/16.pdf

Dean L., Eickholt, C. M., LaFountain, L. J. y Hendrix, K. W. (2020). Effects of maturity on the development of oleic acid and linoleic acid in the four peanut market types. Journal of Food Research, 9(4), 1-9. https://doi. org/10.5539/jfr.v9n4p1

Di Rienzo, J. A., Guzman, A. W. y Casanoves, F. A. (2002). Multiple-comparisons method based on the distribution of the root node distance of a binary tree. Journal of Agricultural, Biological, and Environmental Statistics volume, 7, 129-142. https:// doi.org/10.1198/10857110260141193

Di Rienzo, J., Casanoves, F., González, L., Tablada, M., Robledo C. y Balzarini, M. Infostat. (Versión 2019) [Software]. Córdoba, Argentina: Grupo InfoStat, FCA, Universidad Nacional de Córdoba. URL: http://www. infostat.com.ar

Ellis, M. B. (1971). Dematiaceous Hyphomycetes. Commonwealth Mycological Institute.

Gamba, J. M., Grimoldi, A. S. y Pérez, M. A. (2014). Fenología, rendimiento y tamaño de grano de tres variedades comerciales de maní (Arachis hypogaea L.) en condiciones de campo para la zona central de Córdoba, Argentina. Agriscientia, 31(1), 25-33. https:// doi.org/10.31047/1668.298x.v31.n1.9837

Gamba, J., Sebastián y Pérez, M., Perricone, L., Illa, C. y Pérez, M. A. (2020). Comportamiento de variedades de maníde diferente ciclo en áreas de expansión de siembra en Córdoba Argentina. South American Sciences, 1(2), e2045. http://dx.doi.org/10.17648/sas. 
v1i2.45

Gilman, D. F. y Smith O. D. (1977). Maximum percentage of mature fruit and associated characters at two intrarow spacings in peanuts. Crop Science, 17(4),587-591.

https://doi.org/10.2135/cropsci1977.0011183X00170004 0026x

Gulluoglu, L., Bakal, H., Onat, B., Kurt, C. y Arioglu, $H$. (2016). The effect of harvesting dates on some agronomic and quality characteristics of peanut grown in the Mediterranean Region of Turkey. Turkish Journal of Field Crops, 21(2), 224-232. http://doi.org/ 10.17557/tjfc.20186

Hassan, F. U. y Ahmed, M. (2012). Oil and fatty acid composition of peanut cultivars grown in Pakistan. Pakistan Journal of Botany, 44(2), 627-630.

Jordan, D. L., Danesha, S. y Johnson, D. (2016). Response of the peanut (Arachis hypogaea L.) cultivar Gregory to interactions of digging date and disease management. Advances in Agriculture, 1-9. http:// dx.doi.org/10.1155/2016/5839090

Kaba, J. S., Ofori, K. y Kumaga, F. K. (2014). Interrelationships of yield and components of yield at different stages of maturity in three groundnut (Arachis hypogaea L.) varieties. International Journal of Life Science Research, 2(1), 43-48.

Mathur, S. B. y Kongsdal, O. (2003). Common laboratory seed health testing methods for detecting fungi. International Seed Testing Association.

Mendonça Kluczkovski, A. (2019). Fungal and mycotoxin problems in the nut industry. Current Opinion in Food Science, 29, 56-63. https://doi.org/10.1016/j. cofs.2019.07.009

Onemli, F. (2012). Impact of climate change on oil fatty acid composition of peanut (Arachis hypogaeaL.) in three market classes. Chilean Journal of Agricultural Research, 72(4), 483-488. http://biblioteca.inia.cl/ medios/biblioteca/agritec/NR38748.pdf

Pereira dos Santos, E., Pereira da Silva, R., Scabello Bertonha, R., de Freitas Noronha, R. H. y Zerbato C. (2013). Produtividade e perdas de amendoim em cinco diferentes datas de arranquio. Revista Ciência
Agronômica, 44(4), 695-702. http://www.redalyc.org/ articulo.oa? $\mathrm{id}=195328130005$

Pérez, M. A., Cavallo, A. R. y Pedelini, R. (2004). Indicadores de madurez en frutos de maní (Arachis hypogaea L.) cv. Florman, para la producción de semillas en la provincia de Córdoba, Argentina. Agriscientia, 21(2),77- 83. https://doi.org/10.31047/1668.298x.v21. $\mathrm{n} 2.2670$

Pérez, M. A., Cavallo, A. R. y De Souza Maia, M. (2007). Nivel de infección fúngica natural en relación a la calidad de semillas de maní (Arachis hypogaea L.). Revista Brasileira de Sementes, 29(2), 53-59. https:// dx.doi.org/10.1590/S0101-31222007000200008

Rowland, D. L., Sorensen, R. B., Butts, C. L. y Faircloth, W. H. (2006). Determination of maturity and degree day indices and their success in predicting peanut maturity. Peanut Science. 33(2), 125-136. https://doi. org/10.3146/0095-3679(2006)33[125:DOMADD]2.0. $\mathrm{CO} ; 2$

Ryan, L. C. (2011). Calidad nutricional en variedades de maní tegua y granoleico: estabilidad y aceptabilidad de sus aceites; efecto de su ingesta sobre niveles de lípidos plasmáticos en ratones. Tesis de doctorado no publicada, Facultad de Ciencias Médicas, Universidad Nacional de Córdoba. http://bases.bireme.br/cgi-bin/ wxislind.exe/iah/online/?IsisScript=iah/iah.xis\&src=go ogle\&base $=$ LILACS\&lang $=p \&$ nextAction $=$ Ink\&exprSe arch $=607741 \&$ indexSearch $=$ ID

Young, J. H., Person, N. K., Donald, J. O. y Mayfield, W. D. (1982). Harvesting, curing and energy utilization. En Pattee, H. E. y Young C. T. (Eds.), Peanut Science and Technology (458- 485). American Peanut Research and Education Society.

Williams, J. H. (2000). The implications and applications of resource capture concepts to crop improvement by plant breeding. Agricultural and Forest Meteorology, 104, 49-58.

Zuza, E., Muitia, A., Amane, M., Brandenburg, R. y Mondjana, A. (2017). Effect of harvesting time on groundnut yield and yield components in Northern Mozambique. Journal of Postharvest Technology, 5(2), 55-63. 